\title{
Robust and powerful tests for nonlinear deterministic components*
}

\author{
Sam Astill $^{a}$, David I. Harvey ${ }^{b}$, Stephen J. Leybourne ${ }^{b}$ and A.M. Robert Taylor ${ }^{c}$ \\ a. Department of Economics, University of Warwick. \\ b. Granger Centre for Time Series Econometrics and School of Economics, University of Nottingham. \\ c. Essex Business School, University of Essex.
}

May 2014

\begin{abstract}
We develop a test for the presence of nonlinear deterministic components in a univariate time series, approximated using a Fourier series expansion, designed to be asymptotically robust to the order of integration of the process and to any weak dependence present. Our approach is motivated by the Wald-based testing procedure of Harvey, Leybourne and Xiao (2010) [Journal of Time Series Analysis, vol. 31, p.379-391], but uses a function of an auxiliary unit root statistic to select between the asymptotic $\mathrm{I}(0)$ and $\mathrm{I}(1)$ critical values, rather than modifying the Wald test statistic as in Harvey et al.. We show that our proposed test has uniformly greater local asymptotic power than the test of Harvey et al. when the shocks are I(1), identical local asymptotic power when the shocks are $\mathrm{I}(0)$, and also improved finite sample properties. We also consider the issue of determining the number of Fourier frequencies used to specify any nonlinear deterministic components, evaluating the performance of algorithmic- and information criterion-based model selection procedures.
\end{abstract}

Keywords: Trend function testing; Robust tests; Fourier approximation.

JEL Classification: C22.

\section{Introduction}

The ability to detect the presence and magnitude of deterministic components in a financial or economic time series is of key importance when conducting empirical analysis, particularly for the purposes of forecasting and testing for a unit root. For example, in the latter case, failure to correctly specify a relevant deterministic component present in the data is known to result in non-similar and (usually) inconsistent tests. Moreover, the power of unit root tests to reject the null under the $\mathrm{I}(0)$ alternative when deterministic components are unnecessarily included in a model specification is also reduced.

Traditionally, attention in the literature has focused on a linear deterministic component, most often the case of a constant and/or linear trend. The possibility of breaks in such linear deterministics

\footnotetext{
${ }^{*}$ We are grateful to the Co-Editor, Debopam Bhattacharya, and two anonymous referees for their helpful and constructive comments on an earlier draft. Address correspondence to: Robert Taylor, Essex Business School, University of Essex, Colchester, CO4 3SQ, UK. E-mail: rtaylor@essex.ac.uk
} 
has also received considerable attention, due in particular to the effect these breaks have on standard unit root and stationarity tests; see, inter alia, Perron (1998). However, as noted by Harvey, Leybourne and Xiao (2010) [HLX hereafter], these may not necessarily take the form of an instantaneous break in the trend function. They note that changes in economic aggregates are affected by the response of a large number of potentially heterogeneous individuals who are unlikely to respond instantaneously to shocks. As a consequence, they suggest that a smoothly evolving nonlinear deterministic component might provide a better approximation to the underlying deterministic component of these aggregates.

One possible method to capture such nonlinear behaviour is to approximate the deterministic component using a Fourier series expansion. This approach is explored by Becker et al. (2004) and is found to provide a good approximation for a variety of functions. Enders and Lee (2012) show that modelling the deterministic components of an economic time series via a Fourier function can approximate changes of various forms, such as a number of sharp breaks or deterministic smooth transitions, e.g. exponential or logistic smooth transtions (ESTR or LSTR). Becker et al. (2004) derive a test for the presence of nonlinear deterministic components using a Fourier expansion under the assumption that the shocks are I(0). Enders and Lee (2012) propose a corresponding test under the assumption that the data are $\mathrm{I}(1)$. The practical implementation of these tests is clearly problematic since both assume the order of integration of the data to be known. This results in a circular testing problem as we would need to know the order of integration of our data before performing the tests of either Becker et al. (2004) and Enders and Lee (2012); however, in order to perform a unit root or stationarity test we would need to specify the form of the deterministic component.

Motivated by this problem, and drawing on the robust linear trend tests of Vogelsang (1998), HLX suggest a test that is robust to the order of integration of the data, thereby eliminating this circular testing problem. This is achieved by using a composite statistic based around a Wald statistic (that has a well defined limit distribution for both $\mathrm{I}(0)$ and $\mathrm{I}(1)$ shocks) multiplied by a function of an auxiliary unit root test statistic. This function is specified such that when the shocks are $\mathrm{I}(0)$ it converges in probability to one, leaving the asymptotic distribution of the Wald statistic unaffected, but when the shocks are I(1), it converges to a well-defined limit distribution. Judicious choice of the precise function to be used then allows the asymptotic critical values of the composite test statistic to be lined up in the $\mathrm{I}(0)$ and $\mathrm{I}(1)$ environments, for a given significance level. This approach therefore yields tests that display correct asymptotic size for both $\mathrm{I}(0)$ and $\mathrm{I}(1)$ shocks.

Here we propose an alternative to the methodology of HLX. In our approach a function of an auxiliary unit root test statistic is used to select between the asymptotic $\mathrm{I}(0)$ and $\mathrm{I}(1)$ critical values for the Wald test, rather than creating a composite test via multiplication with the Wald statistic as in HLX. The motivation underlying this is that the presence of the multiplicative function of a unit root test statistic in the HLX procedure impacts negatively on the local asymptotic power when the shocks are I(1), relative to a test that compares the unmodified Wald statistic with its asymptotic I(1) critical value directly. In contrast, the approach considered in this paper always uses the Wald statistic without modification, and ensures that the correct asymptotic critical value is used, appropriate to the order of integration. We show that this new procedure achieves the same local asymptotic power 
as the HLX test in the $\mathrm{I}(0)$ setting, while delivering (often substantial) local asymptotic power gains for I(1) shocks. The new approach also provides improved finite sample behaviour.

The paper is organized as follows. Section 2 outlines the model and the hypotheses of interest, with the HLX procedure reviewed in section 3. Section 4 presents our new approach, with the limiting null distribution and local asymptotic power of the tests detailed in section 5. Section 6 addresses issues related to the practical implementation of the new procedure, and section 7 reports results of Monte Carlo simulations to assess the finite sample properties of the proposed tests for a given number of frequencies in the Fourier expansion. In section 8 we examine the issue of selecting the number of frequencies to include in the Fourier expansion, and compare the performance of a selection algorithm based on the proposed tests with one based on the tests of HLX and also an information criterion approach. Section 9 reports results from an empirical study where the procedures considered in this paper are applied to a number of financial volatility indices, while section 10 concludes.

\section{The Model and Testing Hypotheses}

Following HLX, we consider a sample of $T$ observations generated according to the following data generating process (DGP)

$$
y_{t}=d_{t}+\sum_{f=1}^{n} \gamma_{1 f, T} \sin \left(\frac{2 \pi f t}{T}\right)+\sum_{f=1}^{n} \gamma_{2 f, T} \cos \left(\frac{2 \pi f t}{T}\right)+u_{t}, \quad t=1, \ldots, T .
$$

The nonlinear deterministic component of $y_{t}$ is specified using a Fourier series expansion with the maximum number of frequencies contained in the expansion denoted by $n$, and $f \in Z^{+}$denoting a particular frequency. A linear deterministic component is contained in $d_{t}$, the two leading cases of which are $d_{t}=\alpha$ (a constant) and $d_{t}=\alpha+\beta t$ (a constant and a linear trend).

We allow the stochastic component, $u_{t}$, to be either $\mathrm{I}(0)$ or $\mathrm{I}(1)$, satisfying either Assumption $\mathrm{I}(0)$ or Assumption I(1), respectively, below.

Assumption $\mathbf{I}(\mathbf{0})$ The stochastic process $u_{t}$ is such that $u_{t}=v_{t}$, where the process $\left\{v_{t}\right\}$ satisfies $v_{t}=C(L) \varepsilon_{t}, \quad C(L):=\sum_{i=0}^{\infty} C_{i} L^{i}, \quad C(1)^{2}>0$ with $\sum_{i=0}^{\infty} i\left|C_{i}\right|<\infty$, and where $\varepsilon_{t}$ is a mean zero i.i.d. sequence with $E\left(\varepsilon_{t}^{2}\right)=\sigma_{\varepsilon}^{2}$ and finite fourth moment. The long run variance of $v_{t}$ is defined as $\omega_{v}^{2}:=\lim _{T \rightarrow \infty} T^{-1} E\left(\sum_{t=1}^{T} v_{t}\right)^{2}=\sigma_{\varepsilon}^{2} C(1)^{2}$.

Assumption I(0) ensures that we can apply a Functional Central Limit Theorem (FCLT) to the partial sums of $\left\{v_{t}\right\}$; i.e., $T^{-1 / 2} \sum_{t=1}^{\lfloor r T\rfloor} v_{t} \stackrel{d}{\rightarrow} \omega_{v} W(r)$ where $\lfloor$.$\rfloor denotes the integer part of its argument, \stackrel{d}{\rightarrow}$ denotes weak convergence and $W(r)$ is a standard Wiener process. ${ }^{1}$ When the process is $\mathrm{I}(0)$ we make the following assumption on the Fourier coefficients, $\gamma_{1 f, T}:=\gamma_{1 f} \omega_{v} T^{-1 / 2}, \gamma_{2 f, T}:=\gamma_{2 f} \omega_{v} T^{-1 / 2}$, $f=1, \ldots, n$. The $T^{-1 / 2}$-scalings on the Fourier coefficients are analytical devices that provide the appropriate Pitman drifts for the local asymptotic power analysis that follows. The scaling by $\omega_{v}$ is a

\footnotetext{
${ }^{1}$ We maintain the assumption that $\varepsilon_{t}$ is i.i.d. for consistency with the assumptions in HLX, however, the asymptotic results that follow would continue to hold if we relax this assumption to allow $\varepsilon_{t}$ to be a martingale difference sequence.
} 
convenience device that allows the long run variance to be factored out of the limit distributions that arise for the test statistics outlined in this paper.

Assumption $\mathbf{I}(\mathbf{1})$ The stochastic process $u_{t}$ is such that $\Delta u_{t}=v_{t}$, where the process $\left\{v_{t}\right\}$ is as defined in Assumption I(0).

When the process is I(1) we assume that the Fourier coefficients satisfy, $\gamma_{1 f, T}:=\gamma_{1 f} \omega_{v} T^{1 / 2}, \gamma_{2 f, T}:=$ $\gamma_{2 f} \omega_{v} T^{1 / 2}, f=1, \ldots, n$, with the $T^{1 / 2}$-scalings now representing the appropriate Pitman drifts.

In the context of (1), when testing for the presence of nonlinear deterministic components our null and alternative hypotheses are given by

$$
\begin{aligned}
& H_{0}: \gamma_{1 f, T}=\gamma_{2 f, T}=0, \quad f=1, \ldots, n \\
& H_{1}: \text { at least one of } \gamma_{1 f, T}, \gamma_{2 f, T} \neq 0, \quad f=1, \ldots, n .
\end{aligned}
$$

Under $H_{0}$ the deterministic component in (1) reduces to $d_{t}$, while $H_{1}$ specifies that some form of nonlinear deterministic component is present in the data. The formulation of $H_{1}$ allows for the case where $\gamma_{1 f, T}=\gamma_{2 f, T}=0$ for some but not all frequencies $f=1, \ldots, n$.

\section{The HLX Test}

In order to construct tests that are robust to whether the shocks are I(0) or I(1), HLX first consider the partially summed counterpart to regression (1)

$$
z_{t}=\sum_{s=1}^{t} d_{s}+\sum_{f=1}^{n} \gamma_{1 f, T} \sum_{s=1}^{t} \sin \left(\frac{2 \pi f s}{T}\right)+\sum_{f=1}^{n} \gamma_{2 f, T} \sum_{s=1}^{t} \cos \left(\frac{2 \pi f s}{T}\right)+\eta_{t}
$$

where $z_{t}:=\sum_{s=1}^{t} y_{s}$ and $\eta_{t}:=\sum_{s=1}^{t} u_{s}$. They then consider a scaled Wald statistic for $H_{0}$ against $H_{1}$ based on (2), of the form $S W_{0}^{n}:=\left(R S S_{R}-R S S_{U}\right) / R S S_{U}$, where $R S S_{R}$ denotes the residual sum of squares from OLS regression of $z_{t}$ on $\sum_{s=1}^{t} d_{s}$, and $R S S_{U}$ denotes the residual sum of squares from OLS estimation of the unrestricted regression (2). The notation $S W_{0}^{n}$ indicates we are testing the null of zero frequencies against the alternative of up to $n$ frequencies.

The limit distribution of $S W_{0}^{n}$ depends on whether Assumption I(0) or Assumption I(1) holds, but crucially it is well defined in each case. Consequently, HLX propose a Vogelsang (1998)-type modification, basing their recommended test on the modified statistic $M W_{0}^{n}:=S W_{0}^{n} \exp \left(-b_{\xi}|D F|^{-1}\right)$, where $b_{\xi}$ is a finite positive constant and $D F$ is the Dickey-Fuller $t$-statistic applied to the residuals $\hat{u}_{t}$ obtained from OLS estimation of (1), i.e. $D F=(\hat{\phi}-1) /$ s.e. $(\hat{\phi})$ with $\hat{\phi}$ obtained from the OLS regression

$$
\hat{u}_{t}=\phi \hat{u}_{t-1}+\sum_{i=0}^{p} \zeta_{i} \Delta \hat{u}_{t-i}+e_{t} .
$$

The $|D F|^{-1}$ statistic has the property that it is $O_{p}(1)$ when the shocks are $\mathrm{I}(1)$, since $D F$ has a limit distribution $(U$, say), and converges in probability to zero when the shocks are $\mathrm{I}(0)$. Defining 
the limiting null distributions of $S W_{0}^{n}$ under I(0) and I(1) shocks as $D_{0}$ and $D_{1}$, respectively, it then follows that, under $H_{0}, M W_{0}^{n} \stackrel{d}{\rightarrow} D_{0}$ under Assumption $\mathrm{I}(0)$ and $M W_{0}^{n} \stackrel{d}{\rightarrow} D_{1} \exp \left(-b_{\xi}|U|^{-1}\right)$ under Assumption I(1). The constant $b_{\xi}$ is chosen such that, for a given significance level $\xi$, the $M W_{0}^{n}$ test has the same asymptotic critical value under $\mathrm{I}(0)$ and $\mathrm{I}(1)$ shocks. As a result, the $M W_{0}^{n}$ test will have the correct size asymptotically regardless of whether Assumption I(0) or Assumption I(1) holds.

\section{An Alternative Robust Test}

Our proposed testing procedure involves utilising the same scaled Wald statistic as HLX, namely $S W_{0}^{n}$, but we propose using an auxiliary unit root statistic (denoted by $J$ ) to switch between the critical values appropriate for $S W_{0}^{n}$ under Assumption I(0) and Assumption I(1), i.e. critical values from $D_{0}$ and $D_{1}$, respectively, rather than modifying the test statistic itself with the multiplicative term $\exp \left(-b_{\xi}|D F|^{-1}\right)$ as in HLX. Such an approach has the advantage that it leads to a test with local asymptotic power identical to the tests of HLX when the shocks are I(0), but with greater local asymptotic power when the shocks are I(1) due to the removal of the influence of the auxiliary unit root test statistic on the asymptotic distribution of the test in the $\mathrm{I}(1)$ case.

Denoting by $c v_{0, \xi}$ and $c v_{1, \xi}$ the $\xi$-level critical values from $D_{0}$ and $D_{1}$, respectively, we wish to compare $S W_{0}^{n}$ with $c v_{0, \xi}$ when the shocks are $\mathrm{I}(0)$, and $S W_{0}^{n}$ with $c v_{1, \xi}$ when the shocks are I(1). To achieve this, we consider the following adaptive critical value

$$
c v_{\xi}^{\lambda}:=\lambda_{J, \xi} c v_{0, \xi}+\left(1-\lambda_{J, \xi}\right) c v_{1, \xi}, \quad \lambda_{J, \xi}:=\exp \left(-\tau_{\xi} T^{\delta} J\right)
$$

with $\tau_{\xi}$ and $\delta$ positive constants. We require a value of $\delta$ and a statistic $J$ such that $T^{\delta} J$ converges in probability to zero under Assumption $\mathrm{I}(0)$ but diverges to positive infinity under Assumption $\mathrm{I}(1)$. Then, when the shocks are $\mathrm{I}(0), \lambda_{J, \xi} \stackrel{p}{\rightarrow} 1$ and $c v_{\xi}^{\lambda} \stackrel{p}{\rightarrow} c v_{0, \xi}$, and when the shocks are $\mathrm{I}(1), \lambda_{J, \xi} \stackrel{p}{\rightarrow} 0$ and $c v_{\xi}^{\lambda} \stackrel{p}{\rightarrow} c v_{1, \xi}$. This ensures that the correct critical value is used asymptotically, yielding a robust testing approach. Note that although the constant $\tau_{\xi}$ will play a role in the finite sample behaviour of $\lambda_{J, \xi}$, it is asymptotically irrelevant. In what follows, we denote by $A S W_{0}^{n}$ our proposed test that compares the statistic $S W_{0}^{n}$ with the adaptive critical value $c v_{\xi}^{\lambda}$, given suitable choices of $\delta$ and $J$ that satisfy our requirements on $T^{\delta} J$.

\section{Asymptotic Results}

Defining $\gamma:=\left[\gamma_{11}, \ldots, \gamma_{1 n}, \gamma_{21}, \ldots, \gamma_{2 n}\right]^{\prime}, X_{k}(r):=\left[m_{11}(r), \ldots, m_{1 k}(r), m_{21}(r), \ldots, m_{2 k}(r)\right]^{\prime}$, and $H_{k}(r):=$ $[F(r), \sin (2 \pi r), \ldots, \sin (2 \pi k r), \cos (2 \pi r), \ldots, \cos (2 \pi k r)]^{\prime}$, where $F(r):=1$ if $d_{t}=\alpha, F(r):=(1, r)$ if $d_{t}=\alpha+\beta t, m_{1 f}(r):=\frac{1}{2 \pi f}(1-\cos (2 \pi f r))$ and $m_{2 f}(r):=\frac{1}{2 \pi f} \sin (2 \pi f r)$, the following large sample results obtain (see HLX for the limits of $M W_{0}^{n}$; the limits for $S W_{0}^{n}$ follow in a straightforward way). If $y_{t}$ is generated by (1) under $H_{1}$, then

$$
\text { (a) Under Assumption I(0), } S W_{0}^{n}, M W_{0}^{n} \stackrel{d}{\rightarrow} \frac{\int_{0}^{1} L_{R}(r, \gamma)^{2} d r}{\int_{0}^{1} L_{U}(r)^{2}}-1=: D_{0}(\gamma)
$$


where $L_{R}(r, \gamma)$ denotes the continuous time residuals from the projection of $\gamma^{\prime} X_{n}(r)+W(r)$ onto the space spanned by $\int_{0}^{r} F(s) d s$, and $L_{U}(r)$ denotes the continuous time residuals from the projection of $W(r)$ onto the space spanned by $\left[\int_{0}^{r} F(s), X_{n}(r)^{\prime}\right]$; and

$$
\begin{aligned}
& \text { (b) Under Assumption I(1), } S W_{0}^{n} \stackrel{d}{\rightarrow} \frac{\int_{0}^{1} N_{R}(r, \gamma)^{2} d r}{\int_{0}^{1} N_{U}(r)^{2}}-1=: D_{1}(\gamma) \\
& M W_{0}^{n} \stackrel{d}{\rightarrow} D_{1}(\gamma) \exp \left(-b_{\xi}|U|^{-1}\right)
\end{aligned}
$$

where $N_{R}(r, \gamma)$ denotes the continuous time residuals from the projection of $\boldsymbol{\gamma}^{\prime} X_{n}(r)+\int_{0}^{r} W(s) d s$ onto the space spanned by $\int_{0}^{r} F(s) d s, N_{U}(r)$ denotes the continuous time residuals from the projection of $\int_{0}^{r} W(s) d s$ onto the space spanned by $\left[\int_{0}^{r} F(s), X_{n}(r)^{\prime}\right]$, and $U:=\left(K(1)^{2}-K(0)^{2}-1\right) /\left(2 \sqrt{\int_{0}^{1} K(r)^{2} d r}\right)$, with $K(r)$ the continuous time residuals from the projection of $W(r)$ onto the space spanned by $H_{n}(r)^{\prime}$.

The asymptotic null distributions of $S W_{0}^{n}$ and $M W_{0}^{n}$ obtain by setting $\gamma=0$ in the foregoing representations, so that, linking with the notation of the previous section, $D_{0} \equiv D_{0}(\mathbf{0})$ and $D_{1} \equiv$ $D_{1}(\mathbf{0})$. Note that $D_{0}$ and $D_{1}$ are functions of $n$, and thus the limit distribution of $S W_{0}^{n}$ depends on the choice of $n$. The asymptotic critical values of the with-constant $S W_{0}^{n}\left(d_{t}=\alpha\right)$ and withtrend $S W_{0}^{n}\left(d_{t}=\alpha+\beta t\right)$ statistics, for $n=1,2,3$ and for both $\mathrm{I}(0)$ and $\mathrm{I}(1)$ shocks, are given in Table 1. These were obtained from direct simulation of these limiting distributions, with the Wiener process approximated using $\operatorname{NIID}(0,1)$ random variates, and with the integrals approximated by normalized sums based on 1,000 steps. Here and throughout the paper, all Monte Carlo simulations were conducted in Gauss 9.0 using 50,000 replications. The critical values reported for the I(0) case are also the limit critical values of $M W_{0}^{n}$, and coincide with the values reported in Table 1 of HLX.

Under Assumption I(0), both the $M W_{0}^{n}$ and $A S W_{0}^{n}$ tests share the same asymptotic distribution and, as such, will possess identical local asymptotic power functions. Under Assumption I(1), however, their local asymptotic power functions differ. Henceforth, we will concentrate attention on the withconstant $A S W_{0}^{n}$ test to facilitate direct comparison with the results presented in HLX. Corresponding results for the with-trend variant are available on request. Figure 1 reports the local asymptotic power (at the nominal 0.05 significance level) under both Assumption $\mathrm{I}(0)$ and Assumption $\mathrm{I}(1)$ for $n=1,2,3$, with the results again obtained from direct simulation of the limiting distributions. Due to the multi-parameter nature of the testing problem we present results under the alternative that $\gamma_{11}=\ldots=\gamma_{1 n}=\gamma_{21}=\ldots=\gamma_{2 n}=\gamma$. Panels (a), (c) and (e) confirm that the local asymptotic powers of both tests are identical when the shocks are $\mathrm{I}(0)$. However, when the shocks are I(1), Panels (b), (d) and (f) reveal a distinct power ordering between the two test procedures across all values of $n$. The new $A S W_{0}^{n}$ test has uniformly superior local asymptotic power to the $M W_{0}^{n}$ test, with the potential power gains being quite substantial. Indeed, we find that the maximum power gains across $\gamma$ afforded by using $A S W_{0}^{n}$ rather than $M W_{0}^{n}$ are $0.28,0.30$ and 0.31 for $n=1, n=2$ and $n=3$, respectively. 


\section{Practical Implementation of the Test Procedure}

To implement the $A S W_{0}^{n}$ test, we require choices for $\delta$ and $J$ which satisfy the conditions on $T^{\delta} J$ given below (4). We followed HLX and experimented with functions of the Dickey-Fuller-type statistic, $D F$, and also a Breitung (2002)-type variance ratio unit root statistic

$$
B:=s_{u}^{-2} T^{-3} \sum_{t=1}^{T}\left(\sum_{s=1}^{t} \hat{u}_{s}\right)^{2}
$$

where $s_{u}^{2}:=T^{-1} \sum_{t=1}^{T} \hat{u}_{t}^{2}$, with $\hat{u}_{t}$ the residuals obtained from OLS estimation of (1). While all choices of $\delta$ and $J$ that satisfy the required conditions on $T^{\delta} J$ result in asymptotically equivalent test procedures, we found the best overall finite sample behaviour was achieved when setting $\delta=1 / 2$ and $J=B$. With these choices, following results in Breitung (2002), under Assumption $\mathrm{I}(0)$ we find $B=$ $O_{p}\left(T^{-1}\right)$ and so $T^{1 / 2} J=O_{p}\left(T^{-1 / 2}\right)$, while under Assumption $\mathrm{I}(1), B=O_{p}(1)$ with $T^{1 / 2} J=O_{p}\left(T^{1 / 2}\right)$, clearly satisfying the required conditions on $T^{\delta} J$. We recommend these settings for $\delta$ and $J$ in the implementation of the test.

While the adaptive critical value $c v_{\xi}^{\lambda}$ of (4) delivers an appropriate critical value for $S W_{0}^{n}$ asymptotically, we also consider a finite sample adjustment that proves to be beneficial in controlling the new test's size in small samples. Results from unreported simulations showed that the procedure outlined thus far has a tendency to exhibit finite sample over-size when the shocks are I(1), and finite sample under-size when the shocks are $\mathrm{I}(0)$. We therefore consider a modification designed to inflate (deflate) the finite sample critical value in the $\mathrm{I}(1)(\mathrm{I}(0))$ case. Specifically, we consider the following adjusted adaptive critical value in place of (4)

$$
c v_{\xi}^{\lambda, a d j}:=\lambda_{J, \xi}\left(1-\kappa_{\xi} \lambda_{J, \xi} T^{-1 / 2}\right) c v_{0, \xi}+\left(1-\lambda_{J, \xi}\right)\left\{1+\kappa_{\xi}\left(1-\lambda_{J, \xi}\right) T^{-1 / 2}\right\} c v_{1, \xi}
$$

where $\kappa_{\xi}>0$. Note that this has no effect asymptotically as $\kappa_{\xi} \lambda_{J, \xi} T^{-1 / 2} \stackrel{p}{\rightarrow} 0$ under $\mathrm{I}(0)$ and $\kappa_{\xi}\left(1-\lambda_{J, \xi}\right) T^{-1 / 2} \stackrel{p}{\rightarrow} 0$ under I(1).

Although $\kappa_{\xi}$ and $\tau_{\xi}$ in (6) and (4) have no impact on the asymptotic behaviour of $A S W_{0}^{n}$, specific values of these parameters are required to implement the test in practice, and the choice of these values affects the test's finite sample properties. We calibrated these choices according to a set of unreported size and power simulations for $n=1,2,3$. As a starting point, for a given $n$, we restricted attention to the set of pairings of $\left(\kappa_{\xi}, \tau_{\xi}\right)$ which delivered a correct empirical size of exactly $\xi$ (for each of $\xi=0.10,0.05$ and 0.01$)$ for the pure unit root case $\Delta y_{t}=\varepsilon_{t} \sim N I I D(0,1), y_{1}=\varepsilon_{1}$, and for the sample size $T=300$. Among such pairings, increases in $\tau_{\xi}$ (with corresponding decreases in $\kappa_{\xi}$ ) were found to reduce the degree of any over-size displayed in stationary scenarios, but at the cost of decreased finite sample power. We first chose $\left(\kappa_{\xi}, \tau_{\xi}\right)$ such that the test also had empirical size equal to $\xi$ when $y_{t}=\varepsilon_{t} \sim \operatorname{NIID}(0,1)$ and $T=300$. In some cases, however, we found that this choice led to significant size distortions for moving average $\varepsilon_{t}$; in such cases, we selected a $\left(\kappa_{\xi}, \tau_{\xi}\right)$ pairing with a larger $\tau_{\xi}$ (and therefore smaller $\kappa_{\xi}$ ) to reduce the size distortions. Specifically, we chose a pairing such that, for $T=300$, the empirical size lay below 0.07 for nominal 0.05 -level tests (and below 0.14 and 
0.014 for nominal 0.10 - and 0.01-level tests, respectively) across the $\operatorname{ARMA}(1,1)$ and $\operatorname{ARIMA}(0,1,1)$ simulation settings considered in section 7.1 below. We advocate tolerating this modest amount of potential over-size in order to preserve decent finite sample power levels. The chosen pairings are given in Table 2, and we recommend these settings for practical applications of the test.

\section{$7 \quad$ Finite Sample Simulations}

\subsection{Empirical Size}

In this section we consider the finite sample size behaviour of the $M W_{0}^{n}$ and $A S W_{0}^{n}$ tests, focusing on the case $n=1$. We generate data according to the following DGP, which allows for stationary $\operatorname{ARMA}(1,1)$ and integrated $\operatorname{ARIMA}(0,1,1)$ shocks: $y_{t}=\phi y_{t-1}+\varepsilon_{t}-\theta \varepsilon_{t-1}, t=2, \ldots, T$, with $u_{1}=\varepsilon_{1}$ and $\varepsilon_{t} \sim N I I D(0,1)$. Table 3 reports the empirical sizes of nominal 0.05 -level $M W_{0}^{1}$ and $A S W_{0}^{1}$ tests for the sample sizes $T=\{150,300\}$ and serial correlation parameters $\phi=\{0,0.5,0.7,0.9,0.95,1\}$ and $\theta=\{-0.5,0,0.5\}$. When calculating the $D F$ unit root test statistic required for the $M W_{0}^{1}$ procedure, the lag truncation parameter $p$ in (3) was chosen using the modified Akaike information criterion (MAIC) of Ng and Perron (2001) with $p_{\max }=\left\lfloor 12(T / 100)^{1 / 4}\right\rfloor$, and using the modification of Perron and Qu (2007), as outlined in HLX.

The finite sample sizes of the two tests follow broadly similar patterns across the different serial correlation parameter settings. Both are close to nominal size for $\mathrm{I}(1)$ shocks $(\phi=1)$, apart from some over-size observed for $A S W_{0}^{1}$ when $T=150$. At the other extreme, when $\phi=0$, we see that in the absence of moving average components $(\theta=0)$, the with-constant $A S W_{0}^{1}$ test is approximately correctly sized and the with-trend $A S W_{0}^{1}$ test is a little under-sized, while some modest size distortions are observed when $\theta \neq 0$. On the other hand, the $M W_{0}^{1}$ tests are severely under-sized in all cases when $\phi=0$. For stationary but autocorrelated DGPs $(0<\phi<1)$ all tests can be substantially under-sized in finite samples. As was observed by HLX, for some values of $\phi$ the tests are more under-sized for $T=300$ than for $T=150$; unreported simulations confirm, however, that this phenomenon eventually vanishes for much larger sample sizes, in line with our asymptotic results. The results of Table 3 show that the new $A S W_{0}^{n}$ tests are generally conservative, and are therefore unlikely to spuriously signal the presence of nonlinear deterministic components when they are in fact absent. Furthermore, while under-size is apparent for stationary shocks, the degree of this downward size distortion is less marked for the $A S W_{0}^{n}$ tests than for the $M W_{0}^{n}$ tests.

\subsection{Empirical Power}

To examine the finite sample power properties of the tests, we generate data according to the DGP

$$
\begin{aligned}
& y_{t}=\gamma \sum_{f=1}^{n} \sin \left(\frac{2 \pi f t}{T}\right)+\gamma \sum_{f=1}^{n} \cos \left(\frac{2 \pi f t}{T}\right)+u_{t}, \quad t=1, \ldots, T \\
& u_{t}=\phi u_{t-1}+\varepsilon_{t}, \quad t=2, \ldots, T
\end{aligned}
$$


with $n=1, u_{1}=\varepsilon_{1}$ and $\varepsilon_{t} \sim N I I D(0,1)$. Figure 2 presents power curves for nominal 0.05-level withconstant $M W_{0}^{1}$ and $A S W_{0}^{1}$ tests, for $T=150$ and $\phi=\{0,0.5,0.7,0.9,0.95,1\}$. The power curves were computed using a grid of 50 steps of values for $\gamma$ from to 0 to $\gamma_{\max }$, with $\gamma_{\max }=\{2,4,8,20,20,100\}$, corresponding to the six values of $\phi$ considered.

Figures 2(f) and 3(f) show that when the shocks are I(1), the power of the new $A S W_{0}^{1}$ test is clearly superior to that of $M W_{0}^{1}$, in line with the asymptotic power results in Figure 1. It is reassuring to see that the power gains observed in the limit are also manifest in finite samples, with quite substantial power advantages available through use of $A S W_{0}^{1}$ in the $\mathrm{I}(1)$ setting. When the shocks are $\mathrm{I}(0)$, the two tests are asymptotically equivalent, although it is to be expected that their power properties will differ in finite samples, particularly given the differential finite sample size results discussed above. Indeed, Figures 2(a)-2(e) show that differences between the $A S W_{0}^{1}$ and $M W_{0}^{1}$ power curves do occur. The main observation is that $A S W_{0}^{1}$ generally outperforms $M W_{0}^{1}$ under $\mathrm{I}(0)$ shocks. The power gains associated with $A S W_{0}^{1}$ are most marked when $\phi=0$, where it is evident that the under-size associated with $M W_{0}^{1}$ in this case has a detrimental impact on power relative to the better sized $A S W_{0}^{1}$ test. A similar, albeit less exaggerated, pattern is seen when $\phi=0.5$, while there is little to choose between the two tests when $\phi=0.7$ and $\phi=0.9$. Finally, when $\phi=0.95$, relative power gains are again displayed by $A S W_{0}^{1}$. In summary, the $A S W_{0}^{1}$ test offers valuable improvements in finite sample power relative to $M W_{0}^{1}$, both in the case of I(1) shocks (as would be expected) and also in the case of $\mathrm{I}(0)$ shocks where the tests are asymptotically equivalent.

As suggested by a referee, an alternative to using $\hat{u}_{t}$ in the construction of $B$ would be to instead use residuals obtained from (1) but with the null hypothesis imposed, i.e. to use residuals from OLS estimation of $y_{t}=d_{t}+u_{t}, t=1, \ldots, T$. It can be shown that $c v_{\xi}^{\lambda, a d j}$ computed using $B$ based on such

restricted residuals has exactly the same asymptotic properties as $c v_{\xi}^{\lambda, a d j}$ in (6) under Assumption $\mathrm{I}(0)$ and Assumption I(1), both under the null and under the respective local alternative hypotheses. Unreported simulations show that this approach tends to be susceptible to a greater degree of undersize than our suggested procedure, while neither procedure dominates the other across $\phi$ in terms of finite sample power. As a result, we do not pursue this alternate approach further here.

\section{Determining The Number Of Frequencies}

The analysis in the previous section assumed that the true maximum number of frequencies, $n$, was known. In practice, however, this setting is unknown and must be specified by the practitioner. Unreported simulation results show that incorrectly specifying the number of frequencies, $n$, to include in the testing procedure has a detrimental effect on the power of all of the tests considered in this paper. For instance, if we choose to perform a test for at most $n+1$ frequencies when the true number of frequencies is $n$ we will sacrifice power due to over-specification in the test procedure; similarly, under-specification of $n$ can result in tests with very low power.

HLX attempt to overcome this problem by presenting an algorithm for determining the number 
of frequencies to include in their testing procedure. This algorithm can also be applied to the new testing approach proposed in this paper. As part of the algorithm, HLX develop a test of the null of at most $m-1$ frequencies versus $m$ frequencies, i.e., within the context of (1),

$$
\begin{aligned}
& H_{0}^{*}: \gamma_{1 m, T}=\gamma_{2 m, T}=0 ; \quad \gamma_{1 f, T}, \gamma_{2 f, T}, f=1, \ldots, m-1 \text { unrestricted } \\
& H_{1}^{*}: \text { at least one of } \gamma_{1 m, T}, \gamma_{2 m, T} \neq 0
\end{aligned}
$$

They recommend the test $M W_{m-1}^{m}:=S W_{m-1}^{m} \exp \left(-b_{\xi}|D F|^{-1}\right)$, where $S W_{m-1}^{m}:=\left(R S S_{R}-R S S_{U}\right) / R S S_{U}$ with $R S S_{R}$ and $R S S_{U}$ the restricted and unrestricted residual sums of squares from OLS estimation of (2) with $n$ replaced by $m-1$ and $m$, respectively, and where $D F$ is the Dickey-Fuller $t$-statistic applied to the OLS residuals from estimation of (1) with $n$ replaced by $m$.

The new approach proposed in this paper can also be used to construct a test of $H_{0}^{*}$ against $H_{1}^{*}$. Specifically, we adopt the same $S W_{m-1}^{m}$ statistic that appears as a component in $M W_{m-1}^{m}$, and compare this statistic with the adjusted adaptive critical value $c v_{\xi}^{\lambda, a d j}$ taking the form of (6), where $\lambda_{J, \xi}$ is as defined in (4) with $\delta=1 / 2$ and $J=B$ where $B$ now denotes the statistic in (5) with $\hat{u}_{t}$ being the residuals from OLS estimation of (1) with $n$ replaced by $m$.

The following large sample results for $S W_{m-1}^{m}$ can be obtained directly from HLX. When $y_{t}$ is generated by (1) under $H_{1}^{*}$,

$$
\text { (a) Under Assumption I(0), } S W_{m-1}^{m} \stackrel{d}{\rightarrow} \frac{\int_{0}^{1} L_{R}^{*}\left(r, \gamma^{*}\right)^{2} d r}{\int_{0}^{1} L_{U}^{*}(r)^{2}}-1=: D_{0}^{*}\left(\boldsymbol{\gamma}^{*}\right)
$$

where $\gamma^{*}:=\left[\gamma_{1 m}, \gamma_{2 m}\right], L_{R}^{*}\left(r, \gamma^{*}\right)$ denotes the continuous time residuals from the projection of $\gamma_{1 m} m_{1 m}(r)+\gamma_{2 m} m_{2 m}(r)+W(r)$ onto the space spanned by $\left[\int_{0}^{r} F(s) d s, X_{m-1}(r)^{\prime}\right]$, and $L_{U}^{*}(r)$ denotes the continuous time residuals from the projection of $W(r)$ onto the space spanned by $\left[\int_{0}^{r} F(s) d s, X_{m}(r)^{\prime}\right]$; and

$$
\text { (b) Under Assumption I(1), } S W_{m-1}^{m} \stackrel{d}{\rightarrow} \frac{\int_{0}^{1} N_{R}^{*}\left(r, \gamma^{*}\right)^{2} d r}{\int_{0}^{1} N_{U}^{*}(r)^{2}}-1=: D_{1}^{*}\left(\gamma^{*}\right)
$$

where $N_{R}^{*}\left(r, \gamma^{*}\right)$ denotes the continuous time residuals from the projection of $\gamma_{1 m} m_{1 m}(r)+\gamma_{2 m} m_{2 m}(r)+$ $\int_{0}^{r} W(s) d s$ onto the space spanned by $\left[\int_{0}^{r} F(s) d s, X_{m-1}(r)^{\prime}\right]$, and $N_{U}^{*}(r)$ denotes the continuous time residuals from the projection of $\int_{0}^{r} W(s) d s$ onto the space spanned by $\left[\int_{0}^{r} F(s) d s, X_{m}(r)^{\prime}\right]$.

The asymptotic distributions under the null $H_{0}^{*}$ follow by setting $\gamma^{*}=0$ in the above limits. Asymptotic critical values for $S W_{m-1}^{m}$ for $m=2,3$ under both Assumption I(0) and Assumption I(1) are given in Table 1. We also determined suitable values for $\tau_{\xi}$ and $\kappa_{\xi}$ to be used in constructing $c v_{\xi}^{\lambda, a d j}$, and these recommended values are given in Table 2 . Hereafter we denote by $A S W_{m-1}^{m}$ the new tests that compare $S W_{m-1}^{m}$ with $c v_{\xi}^{\lambda, a d j}$.

Following the HLX algorithm, the $A S W_{0}^{n}$ and $A S W_{m-1}^{m}$ tests can now be used to determine the number of frequencies, $n$. Given an assumption on the largest possible value of $n, n_{\max }$, we first conduct the tests $A S W_{0}^{i}, i=1, \ldots, n_{\max }$, and if none of these tests reject we conclude $n=0$. If any of the tests do reject, we identify the largest value of $i$ for which the null is rejected and set $m$ to this value. If $m=1$ it is concluded that $n=1$; otherwise if $m>1$ we conclude that $m$ is the largest 
value that $n$ might take. We then consider $A S W_{0}^{m-1}$, and if this test fails to reject we set $n=m$. If, however, $A S W_{0}^{m-1}$ does reject we then perform the $A S W_{m-1}^{m}$ test; then if this test rejects we conclude $n=m$, otherwise we reduce $m$ by one and repeat the loop. For a diagrammatic representation of the algorithm, see Figure 3 of HLX.

A natural alternative approach to identifying the number of frequencies present in a series would be a standard model selection criterion. As such we also consider a Bayesian information criterion (BIC) approach, which for the constant case $\left(d_{t}=\alpha\right)$ is based on the following two regressions

$$
\begin{aligned}
& \Delta y_{t}=\alpha+\rho y_{t-1}+\sum_{f=1}^{n} \gamma_{1 f, T} \sin \left(\frac{2 \pi f t}{T}\right)+\sum_{f=1}^{n} \gamma_{2 f, T} \cos \left(\frac{2 \pi f t}{T}\right)+\sum_{i=0}^{k} c_{i} \Delta y_{t-i}+e_{t} \\
& \Delta y_{t}=\sum_{f=1}^{n} \gamma_{1 f, T}^{*} \sin \left(\frac{2 \pi f t}{T}\right)+\sum_{f=1}^{n} \gamma_{2 f, T}^{*} \cos \left(\frac{2 \pi f t}{T}\right)+\sum_{i=0}^{k} c_{i}^{*} \Delta y_{t-i}+e_{t}^{*} .
\end{aligned}
$$

Consider selecting $n$ by minimising the BIC across $n=\left\{0,1, \ldots, n_{\max }\right\}$ and $k=\left\{0,1, \ldots, k_{\max }\right\}$ for a given regression. BIC based on (9) will be appropriate when the shocks are $\mathrm{I}(0)$, while BIC based on (10) will be appropriate for I(1) shocks. Consequently, we propose minimising the BIC jointly over both regressions (9) and (10), and it this procedure with which we will compare the performance of the HLX-type algorithm below.

We now assess, by means of Monte Carlo simulation, the relative performance of the HLX algorithm based on $M W_{0}^{n}$ and $M W_{m-1}^{m}$ (which we refer to as $M W$ ), the same algorithmic approach based on $A S W_{0}^{n}$ and $A S W_{m-1}^{m}$ (which we denote by $A S W$ ), and the BIC procedure outlined above. Note that all tests were performed for the with-constant variants and were conducted at the nominal 0.05-level. Data were generated according to (7)-(8) with $n=2$. We compute the frequency with which each procedure correctly selects $n=2$, along with the frequencies with which each of the incorrect selections of $n=0,1,3$ are made. The choices $n_{\max }=3$ and $k_{\max }=4$ are adopted in all cases, and we consider experiments with $T=150, \phi=\{0,0.5,0.7,0.9,0.95,1\}$, as before. For each value we report results for four different values of $\gamma$, including the case $\gamma=0$. Results for serially correlated $\varepsilon_{t}$ were found to be similar to those for i.i.d. innovations and are available upon request.

The results are reported in Table 4. Comparing first the two algorithmic approaches, we observe that $A S W$ generally outperforms $M W$, in line with the superior finite sample testing properties that the constituent tests involved in $A S W$ display. Due to the inherent multiple testing issues with the algorithms, when $\gamma=0$ we see that both $A S W$ and $M W$ select a non-zero value of $n$ with frequency greater than the nominal level, less so in the case of $M W$ due to its lower finite sample size. However, as might be expected from the results of section 7, when $\gamma>0$ the $A S W$ approach generally outperforms the $M W$ approach in terms of the frequency with which $n=2$ is selected. Indeed, the improvements offered by $A S W$ over the original $M W$ are quite substantial in a number of cases, particularly for modest values of $\gamma$. While $M W$ can outperform $A S W$, such gains are always relatively minor, and tend to occur in situations where both procedures select $n=2$ with high probability.

Turning our attention to a comparison of $A S W$ with the BIC approach, it can be seen that when $\gamma=0$, BIC selects a value of $n$ greater than zero with roughly the same frequency as was seen for 
$A S W$. In some cases, differences are seen, but neither procedure dominates the other overall when there are truly no nonlinear deterministic components present. When $\gamma>0$, we find that neither $A S W$ nor BIC dominates the other across the different $\gamma$ magnitudes considered. While the performance of the two methods for determining the number of frequencies can be quite different for any given DGP, these differences do not follow a systematic pattern across all $\phi$ or $\gamma$; as such, it is difficult to argue for a particular ranking between $A S W$ and BIC. What is clear, however, is that both procedures offer improvements relative to the $M W$ approach of HLX.

The previous simulation study has examined the ability of the proposed algorithms and BIC procedure to correctly specify $n$ when the deterministic component of the series is exactly approximated by a Fourier series expansion. It is also important to investigate how useful these approaches are in approximating other forms of nonlinear deterministic components. To that end we generated data according to $y_{t}=d_{t}+u_{t}, t=1, \ldots, T$, with $u_{t}$ as in (8) for $\phi=\{0,0.5,1\}$, and with $d_{t}$ specified as either a mid-sample ESTR, i.e. $d_{t}=\gamma\left[1-\exp \left(-0.1(t-0.5 T)^{2}\right)\right]$, or as a mid-sample LSTR, i.e. $d_{t}=\gamma /[1+\exp (0.1(t-0.5 T))]$. For a sample size of $T=150$ we compute the power of the methods to detect nonlinear deterministic elements, measured as the percentage of replications for which the $A S W$ algorithm or BIC selects $n>0$ frequencies. Table 5 reports, for a range of $\gamma$ magnitudes, the power of with-constant $A S W$ and BIC (results are omitted for $M W$ as its performance was uniformly worse than $A S W)$. For ESTR deterministics, both $A S W$ and BIC have power that is increasing in $\gamma$, indicating that the Fourier approximation works reasonably well in terms of modelling the ESTR-type nonlinearities. Of the two procedures, BIC offers higher power than $A S W$, particularly when $\phi>0$.

In the case of LSTR deterministics, however, the powers of both with-constant $A S W$ and BIC are typically decreasing in $\gamma$ (with the exception of BIC when $\phi=1$ ), so while we again find that BIC outperforms $A S W$ here, neither procedure performs well across all values of $\phi$ considered. This feature arises since the LSTR component involves a relatively slow transition from one level to another, which is not well modelled by the Fourier terms of frequency $f=1,2,3$ that are included in the fitted unrestricted model (this approximation error may also be magnified in the $A S W$ procedure due to the statistics being based on the partially summed regression (2)). To capture these low frequency movements, one would need to incorporate Fourier terms with lower frequency than one, e.g. $f=0.5$, in the approximation. Of course, such low frequency Fourier terms can themselves be reasonably well approximated by a linear trend term, hence we also report in Table 5 results obtained from application of the with-trend version of $A S W$ to the LSTR data generating processes. We see that for the cases where the with-constant $A S W$ suffered from very low power, the with-trend variant has decent power which is also now increasing in the magnitude of $\gamma$. We also see in Table 5 that with-trend $A S W$ also delivers some power improvements over with-constant $A S W$ in the ESTR simulations, particularly when $\phi=1$. It appears, therefore, that when low frequency changes are present in the data, the withtrend variant of $A S W$ is a potentially more robust approach for detecting deterministic nonlinearities, as the underlying fitted models provide a superior approximation to the true deterministic component. 


\section{Empirical Application}

There has been much interest in the financial literature in modelling the volatility of economic indicators, most notably financial indicators such as stock market indices. While early work by, inter alia, Poterba and Summers (1986), French et al. (1987) and Schwert and Seguin (1990) concentrated on modelling volatility in stock market indices using linear methods, more recent work by Cao and Tsay (1992) attempts to use nonlinear methods to analyse such indices using threshold autoregressive and nonlinear GARCH and EGARCH models. Such methods assume that any observed nonlinearity in the volatility indices is stochastic rather than deterministic. It is of interest, however, to assess whether we can detect nonlinear behaviour in the deterministic components of such volatility indices.

To that end we collected daily data on six volatility indices for the one year period ending 18 March 2013. Five of these indices measure the volatility of a particular stock market index: the Dow Jones Industrial Average Volatility Index (Ticker: VXD), the NASDAQ-100 Volatility Index (VXN), the S\&P 500 Volatility Index (VIX), the S\&P 100 Volatility Index (VXO) and the FTSE 100 Volatility Index (VFTSE). The final series considered is the EuroCurrency Volatility Index (EVZ), which is an index of the volatility of the US\$/EUR exchange rate. We applied the with-constant $A S W$ and $M W$ algorithms described in section 8 (with $n_{\max }=3$ ), implemented at both the 0.05 and 0.10 significance levels, to each series. We also applied the BIC selection procedure as a point of comparison. The number of frequencies, $\hat{n}$, detected by these methods are reported in Table 6 . In all cases, some form of nonlinear deterministic behaviour is detected in the volatility indices, suggesting a consistent body of evidence for nonlinear behaviour in the deterministic components of these financial volatility series. The pattern of results observed in Table 6 is consistent with the finite sample simulations presented in sections 7 and 8. In all cases, for a given significance level, $A S W$ chooses $\hat{n}$ to be greater than or equal to that chosen by $M W$; indeed there are three series for which $M W$ does not find any evidence of nonlinearity at the 0.05 -level, while $A S W$ selects $\hat{n}=2$. In comparison with the BIC procedure, we find that $A S W$ finds more evidence for deterministic nonlinearity, with BIC selecting $\hat{n}=0$ for four of the series, and never identifying a greater number of frequencies than $A S W$.

As a measure of the underlying persistence in each series, Table 6 also reports (in parentheses) the estimate of $\phi$ obtained from the $D F$ regression (3) used in the $M W_{0}^{n}$ statistic, where $n$ is set to the corresponding number of frequencies listed in Table 6 . These suggest that the series are highly persistent around a nonlinear deterministic component, but it is unclear whether the stochastic components would be best modelled by stationary or unit root processes. This highlights the advantages of the robust procedures considered in this paper, as we do not need to take a stand on the integration properties of the data. Interestingly, fitting only a constant to the data results in estimates of $\phi$ very close to unity, suggesting that failure to specify the nonlinear deterministic components of these series could well lead to the inference that they contain a unit root. Note also that since the with-constant $A S W$ procedure always selects $\hat{n}>0$, it does not appear that problematic low frequency movements are a feature of these time series.

The fitted deterministic components associated with the corresponding values of $\hat{n}$ obtained by 
$A S W$ are presented alongside the original and detrended series in Figure 3. Note that for the EVZ series we plot the fitted and detrended series for $\hat{n}=3$ rather than $\hat{n}=1$. We see that the deterministic components detected by $A S W$ appear to fit the data rather well, with the apparent nonlinear behaviour in the series well approximated by the Fourier frequency representation in most cases.

\section{Conclusions}

We have proposed a new approach to testing for the presence of nonlinear deterministic components in an economic time series designed to be robust to the order of integration of the data and to any weak dependence present. The recommended approach involves using a scaled Wald statistic that has well defined, but different, limit distributions depending on whether the stochastic component of the series is $\mathrm{I}(0)$ or $\mathrm{I}(1)$. Robustness is achieved by using an adaptive critical value constructed so that the appropriate $\mathrm{I}(0)$ or $\mathrm{I}(1)$ critical value is selected asymptotically. This delivers a test with the same asymptotic properties as the test of HLX in an $\mathrm{I}(0)$ environment, but with worthwhile local asymptotic power gains in an I(1) setting. Monte Carlo simulations suggest that the new test also has superior overall finite sample size and power properties to the HLX test for both $\mathrm{I}(0)$ and $\mathrm{I}(1)$ shocks. The proposed testing procedure can be employed in the algorithm of HLX to determine the number of frequencies used to model a series that contains potential nonlinear deterministic components. We have also considered a model selection approach based on the BIC which was also shown to outperform the original HLX procedure. Our empirical application to financial market volatility indices over the last year supports the notion that the new procedures offer benefits over extant methods.

\section{References}

Becker, R., Enders, W. and Hurn, S. (2004). A general test for time dependence in parameters. Journal of Applied Econometrics 19, 899-906.

Becker, R., Enders, W. and Lee, J. (2006). A stationarity test in the presence of an unknown number of smooth breaks. Journal of Time Series Analysis 27, 381-409.

Breitung, J. (2002). Nonparametric tests for unit roots and cointegration. Journal of Econometrics 108, 343-363.

Cao, C.Q. and Tsay, R.S. (1992). Nonlinear time-series analysis of stock volatilities. Journal of Applied Econometrics 7, 165-185.

Enders, W. and Lee, J. (2012). A unit root test using a Fourier series to approximate smooth breaks. Oxford Bulletin of Economics and Statistics 74, 574-599.

French, K.R., Schwert, G.W. and Stambaugh, R. (1987). Expected stock returns and volatility. Journal of Financial Economics 19, 3-29. 
Harvey, D.I., Leybourne, S.J. and Xiao, L. (2010). Testing for nonlinear deterministic components when the order of integration is unknown. Journal of Time Series Analysis 31, 379-391.

Ng, S. and Perron, P. (2001). Lag length selection and the construction of unit root tests with good size and power. Econometrica 69, 1519-1554.

Perron, P. (1998). Trends and random walks in macroeconomic time series: further evidence from a new approach. Journal of Economic Dynamics and Control 12, 297-332.

Perron, P. and Qu, Z. (2007). A simple modification to improve the finite sample properties of $\mathrm{Ng}$ and Perron's unit root tests. Economics Letters 94, 12-19.

Poterba, J.M. and Summers, L.H. (1986). The persistence of volatility and stock market fluctuaions. American Economic Review 76, 1143-1151.

Schwert, G.W. and Seguin, P.J. (1990). Heteroscedasticity in stock returns. Journal of Finance 45, 1129-1155.

Vogelsang, T.J. (1998). Trend function hypothesis testing in the presence of serial correlation. Econometrica $66,123-148$. 
Table 1. Asymptotic $\xi$-level critical values for $S W_{0}^{n}$ and $S W_{m-1}^{m}$ statistics

\begin{tabular}{|c|c|c|c|c|c|c|c|c|c|}
\hline \multirow[b]{2}{*}{$\xi$} & \multicolumn{3}{|c|}{$n=1$} & \multicolumn{3}{|c|}{$n=2$} & \multicolumn{3}{|c|}{$n=3$} \\
\hline & 0.10 & 0.05 & 0.01 & 0.10 & 0.05 & 0.01 & 0.10 & 0.05 & 0.01 \\
\hline & \multicolumn{9}{|c|}{ With-constant $S W_{0}^{n}$} \\
\hline$c v_{0, \xi}$ & 5.268 & 7.439 & 13.370 & 9.337 & 12.754 & 21.495 & 13.259 & 17.702 & 29.159 \\
\hline \multirow[t]{2}{*}{$c v_{1, \xi}$} & 50.631 & 76.222 & 167.163 & 193.162 & 274.977 & 565.278 & 475.264 & 680.520 & 1300.226 \\
\hline & \multicolumn{9}{|c|}{ With-trend $S W_{0}^{n}$} \\
\hline$c v_{0, \xi}$ & 2.677 & 3.708 & 6.370 & 4.685 & 6.124 & 9.921 & 6.550 & 8.468 & 13.308 \\
\hline \multirow[t]{2}{*}{$c v_{1, \xi}$} & 30.601 & 47.342 & 103.816 & 100.248 & 150.228 & 295.168 & 227.125 & 329.713 & 644.469 \\
\hline & \multicolumn{3}{|c|}{$m=2$} & \multicolumn{3}{|c|}{$m=3$} & & & \\
\hline \multirow[t]{2}{*}{$\xi$} & 0.10 & 0.05 & 0.01 & 0.10 & 0.05 & 0.01 & & & \\
\hline & \multicolumn{6}{|c|}{ With-constant $S W_{m-1}^{m}$} & & & \\
\hline$c v_{0, \xi}$ & 1.779 & 2.406 & 4.007 & 1.038 & 1.393 & 2.252 & & & \\
\hline \multirow[t]{2}{*}{$c v_{1, \xi}$} & 9.829 & 14.138 & 27.101 & 4.636 & 6.437 & 12.147 & & & \\
\hline & \multicolumn{6}{|c|}{ With-trend $S W_{m-1}^{m}$} & & & \\
\hline$c v_{0, \xi}$ & 1.310 & 1.774 & 2.904 & 0.856 & 1.138 & 1.849 & & & \\
\hline$c v_{1, \xi}$ & 8.556 & 12.624 & 24.795 & 4.591 & 6.513 & 12.293 & & & \\
\hline
\end{tabular}

Table 2. $\tau_{\xi}$ and $\kappa_{\xi}$ values for $A S W_{0}^{n}$ and $A S W_{m-1}^{m}$ tests

\begin{tabular}{|c|c|c|c|c|c|c|c|c|c|}
\hline \multirow[b]{2}{*}{$\xi$} & \multicolumn{3}{|c|}{$n=1$} & \multicolumn{3}{|c|}{$n=2$} & \multicolumn{3}{|c|}{$n=3$} \\
\hline & 0.10 & 0.05 & 0.01 & 0.10 & 0.05 & 0.01 & 0.10 & 0.05 & 0.01 \\
\hline & \multicolumn{9}{|c|}{ With-constant $A S W_{0}^{n}$} \\
\hline$\tau_{\xi}$ & 14.6 & 17.5 & 26.0 & 23.0 & 29.5 & 50.0 & 32.0 & 41.0 & 90.0 \\
\hline \multirow[t]{2}{*}{$\kappa_{\xi}$} & 5.8 & 6.9 & 8.5 & 10.5 & 11.2 & 8.6 & 14.3 & 14.5 & 6.5 \\
\hline & \multicolumn{9}{|c|}{ With-trend $A S W_{0}^{n}$} \\
\hline$\tau_{\xi}$ & 43.2 & 50.0 & 63.0 & 79.0 & 110.0 & 180.0 & 151.0 & 210.0 & 325.0 \\
\hline \multirow[t]{2}{*}{$\kappa_{\xi}$} & 9.5 & 9.8 & 10.5 & 14.4 & 8.3 & 5.0 & 11.2 & 6.4 & 3.0 \\
\hline & \multicolumn{3}{|c|}{$m=2$} & \multicolumn{3}{|c|}{$m=3$} & & & \\
\hline$\xi$ & 0.10 & 0.05 & 0.01 & 0.10 & 0.05 & 0.01 & & & \\
\hline \multicolumn{10}{|c|}{ With-constant $A S W_{m-1}^{m}$} \\
\hline$\tau_{\xi}$ & 32.4 & 37.0 & 53.9 & 47.7 & 57.0 & 80.0 & & & \\
\hline$\kappa_{\xi}$ & 4.9 & 5.6 & 7.4 & 4.4 & 5.2 & 6.8 & & & \\
\hline \multicolumn{10}{|c|}{ With-trend $A S W_{m-1}^{m}$} \\
\hline$\tau_{\xi}$ & 90.0 & 105.0 & 160.0 & 140.0 & 195.0 & 315.0 & & & \\
\hline$\kappa_{\xi}$ & 9.9 & 8.6 & 5.9 & 10.6 & 6.0 & 2.1 & & & \\
\hline
\end{tabular}


Table 3. Finite sample size of $M W_{0}^{1}$ and $A S W_{0}^{1}$ tests

\begin{tabular}{|c|c|c|c|c|c|c|c|c|c|}
\hline \multirow[b]{3}{*}{$\phi$} & \multirow[b]{3}{*}{$\theta$} & \multicolumn{4}{|c|}{ With-constant tests } & \multicolumn{4}{|c|}{ With-trend tests } \\
\hline & & \multicolumn{2}{|c|}{$T=150$} & \multicolumn{2}{|c|}{$T=300$} & \multicolumn{2}{|c|}{$T=150$} & \multicolumn{2}{|c|}{$T=300$} \\
\hline & & $M W_{0}^{1}$ & $A S W_{0}^{1}$ & $M W_{0}^{1}$ & $A S W_{0}^{1}$ & $M W_{0}^{1}$ & $A S W_{0}^{1}$ & $M W_{0}^{1}$ & $A S W_{0}^{1}$ \\
\hline \multirow[t]{3}{*}{0.00} & -0.5 & 0.006 & 0.033 & 0.010 & 0.034 & 0.002 & 0.010 & 0.004 & 0.014 \\
\hline & 0.0 & 0.009 & 0.051 & 0.012 & 0.050 & 0.004 & 0.026 & 0.007 & 0.032 \\
\hline & 0.5 & 0.004 & 0.057 & 0.011 & 0.063 & 0.001 & 0.053 & 0.006 & 0.068 \\
\hline \multirow[t]{3}{*}{0.50} & -0.5 & 0.003 & 0.018 & 0.004 & 0.018 & 0.000 & 0.003 & 0.001 & 0.004 \\
\hline & 0.0 & 0.007 & 0.025 & 0.008 & 0.024 & 0.002 & 0.007 & 0.003 & 0.007 \\
\hline & 0.5 & 0.009 & 0.051 & 0.012 & 0.050 & 0.004 & 0.026 & 0.007 & 0.032 \\
\hline \multirow[t]{3}{*}{0.70} & -0.5 & 0.002 & 0.014 & 0.003 & 0.011 & 0.000 & 0.002 & 0.001 & 0.002 \\
\hline & 0.0 & 0.006 & 0.018 & 0.006 & 0.014 & 0.002 & 0.004 & 0.002 & 0.003 \\
\hline & 0.5 & 0.008 & 0.040 & 0.006 & 0.033 & 0.004 & 0.015 & 0.003 & 0.013 \\
\hline \multirow{3}{*}{0.90} & -0.5 & 0.010 & 0.022 & 0.005 & 0.009 & 0.002 & 0.005 & 0.001 & 0.001 \\
\hline & 0.0 & 0.015 & 0.025 & 0.007 & 0.010 & 0.005 & 0.006 & 0.002 & 0.002 \\
\hline & 0.5 & 0.015 & 0.045 & 0.007 & 0.018 & 0.006 & 0.017 & 0.002 & 0.005 \\
\hline \multirow[t]{3}{*}{0.95} & -0.5 & 0.024 & 0.041 & 0.011 & 0.015 & 0.008 & 0.014 & 0.003 & 0.003 \\
\hline & 0.0 & 0.030 & 0.044 & 0.013 & 0.016 & 0.013 & 0.016 & 0.005 & 0.004 \\
\hline & 0.5 & 0.031 & 0.069 & 0.014 & 0.025 & 0.015 & 0.032 & 0.005 & 0.008 \\
\hline \multirow[t]{3}{*}{1.00} & -0.5 & 0.039 & 0.058 & 0.040 & 0.049 & 0.038 & 0.057 & 0.039 & 0.049 \\
\hline & 0.0 & 0.047 & 0.060 & 0.047 & 0.050 & 0.047 & 0.061 & 0.047 & 0.050 \\
\hline & 0.5 & 0.049 & 0.076 & 0.048 & 0.058 & 0.052 & 0.091 & 0.049 & 0.063 \\
\hline
\end{tabular}

Table 4. Number of frequencies selected by with-constant $M W$ and $A S W$ algorithms and BIC: $T=150$

\begin{tabular}{|c|c|c|c|c|c|c|c|c|c|c|c|c|c|}
\hline \multirow[b]{2}{*}{$\phi$} & \multirow[b]{2}{*}{$\gamma$} & \multicolumn{4}{|c|}{$M W$} & \multicolumn{4}{|c|}{$A S W$} & \multicolumn{4}{|c|}{ BIC } \\
\hline & & $n=0$ & $n=1$ & $n=2$ & $n=3$ & $n=0$ & $n=1$ & $n=2$ & $n=3$ & $n=0$ & $n=1$ & $n=2$ & $n=3$ \\
\hline \multirow[t]{4}{*}{0.00} & 0.00 & 0.989 & 0.009 & 0.001 & 0.000 & 0.933 & 0.050 & 0.014 & 0.004 & 0.991 & 0.009 & 0.000 & 0.000 \\
\hline & 0.30 & 0.419 & 0.017 & 0.501 & 0.064 & 0.208 & 0.043 & 0.679 & 0.070 & 0.281 & 0.144 & 0.565 & 0.009 \\
\hline & 0.60 & 0.051 & 0.000 & 0.882 & 0.067 & 0.014 & 0.000 & 0.931 & 0.055 & 0.001 & 0.000 & 0.984 & 0.014 \\
\hline & 1.20 & 0.003 & 0.000 & 0.976 & 0.022 & 0.000 & 0.000 & 0.950 & 0.050 & 0.000 & 0.000 & 0.986 & 0.014 \\
\hline \multirow[t]{4}{*}{0.50} & 0.00 & 0.993 & 0.007 & 0.001 & 0.000 & 0.971 & 0.024 & 0.004 & 0.001 & 0.987 & 0.012 & 0.001 & 0.000 \\
\hline & 0.70 & 0.408 & 0.004 & 0.528 & 0.0 & 0.334 & 0.006 & 0.578 & & 0.274 & 0.051 & 0.656 & 0.020 \\
\hline & 1.40 & 0.030 & 0.000 & 0.917 & 0.052 & 0.032 & 0.000 & 0.906 & 0.0 & .009 & 0.000 & 0.964 & 0.027 \\
\hline & 2.80 & 0.001 & 0.000 & 0.982 & 0.017 & 0.000 & 0.000 & 0.955 & 0.045 & 0.000 & 0.000 & 0.973 & 0.027 \\
\hline \multirow[t]{4}{*}{0.70} & 0.00 & 0.993 & 0.006 & 0.001 & 0.00 & 0.978 & 0.017 & 0.004 & 0.001 & 0.981 & 0.016 & 0.002 & 0.001 \\
\hline & 1.20 & 0.450 & 0.002 & 0.481 & 0.06 & 0.383 & 0.002 & 0.514 & 0.1 & 433 & 0.013 & 0.524 & 0.029 \\
\hline & 2.40 & 0.032 & 0.000 & 0.915 & 0.053 & 0.040 & 0.000 & 0.881 & 0.078 & 0.032 & 0.000 & 0.919 & 0.049 \\
\hline & 4.80 & 0.001 & 0.000 & 0.977 & 0.023 & 0.000 & 0.000 & 0.949 & 0.051 & 0.000 & 0.000 & 0.949 & 0.051 \\
\hline \multirow[t]{4}{*}{0.90} & 0.00 & 0.977 & 0.014 & 0.005 & 0.003 & 0.952 & 0.023 & 0.013 & 0.011 & 0.943 & 0.037 & 0.013 & 0.007 \\
\hline & 3.20 & 0.349 & 0.000 & 0.510 & 0.141 & 0.248 & 0.000 & 0.577 & 0.175 & 0.277 & 0.000 & 0.596 & 0.127 \\
\hline & 6.40 & 0.038 & 0.000 & 0.861 & 0.101 & 0.013 & 0.000 & 0.877 & 0.110 & 0.000 & 0.000 & 0.836 & 0.164 \\
\hline & 12.80 & 0.002 & 0.000 & 0.935 & 0.063 & 0.000 & 0.000 & 0.909 & 0.091 & 0.000 & 0.000 & 0.836 & 0.164 \\
\hline \multirow[t]{4}{*}{0.95} & 0.00 & 0.939 & 0.028 & 0.018 & 0.016 & 0.896 & 0.040 & 0.033 & 0.031 & 0.906 & 0.051 & 0.025 & 0.018 \\
\hline & 4.00 & 0.364 & 0.000 & 0460 & 0.167 & 0.260 & 0.000 & & & 0.145 & 0.000 & 0.659 & 0.195 \\
\hline & 8.00 & 0.063 & 0.000 & 0.799 & 0.138 & 0.016 & 0.000 & 0.855 & 0.12 & 0.000 & 0.000 & 0.781 & 0.218 \\
\hline & 16.00 & 0.007 & 0.000 & 0.904 & 0.089 & 0.000 & 0.000 & 0.892 & 0.108 & 0.000 & 0.000 & 0.782 & 0.218 \\
\hline \multirow[t]{4}{*}{1.00} & 0.00 & 0.900 & 0.041 & 0.030 & 0.029 & 0.876 & 0.050 & 0.039 & 0.035 & 0.883 & 0.064 & 0.031 & 0.021 \\
\hline & 10.00 & 0.369 & 0.000 & 0.515 & 0.116 & 0.234 & 0.000 & 0.667 & 0.099 & 0.000 & 0.000 & 0.814 & 0.186 \\
\hline & 20.00 & 0.209 & 0.000 & 0.690 & 0.10 & 0.032 & 0.000 & 0.903 & 0.065 & 0.000 & 0.000 & 0.814 & 0.186 \\
\hline & 40.00 & 0.116 & 0.000 & 0.796 & 0.088 & 0.000 & 0.000 & 0.944 & 0.056 & 0.000 & 0.000 & 0.814 & 0.186 \\
\hline
\end{tabular}


Table 5. Power of $A S W$ algorithm and BIC to detect exponential and logistic smooth transitions: $T=150$

\begin{tabular}{|c|c|c|c|c|c|c|c|c|}
\hline \multirow[b]{2}{*}{$\phi$} & \multicolumn{4}{|c|}{ ESTR } & \multicolumn{4}{|c|}{ LSTR } \\
\hline & $\gamma$ & $A S W_{c}$ & $\mathrm{BIC}$ & $A S W_{t}$ & $\gamma$ & $A S W_{c}$ & BIC & $A S W_{t}$ \\
\hline \multirow[t]{3}{*}{0.0} & 1.0 & 0.508 & 0.526 & 0.520 & 2.0 & 0.484 & 0.969 & 0.263 \\
\hline & 1.5 & 0.806 & 0.929 & 0.837 & 4.0 & 0.055 & 0.428 & 0.687 \\
\hline & 2.0 & 0.936 & 0.997 & 0.958 & 6.0 & 0.001 & 0.034 & 0.895 \\
\hline \multirow[t]{3}{*}{0.5} & 3.0 & 0.588 & 0.893 & 0.660 & 10.0 & 0.001 & 0.042 & 0.474 \\
\hline & 4.0 & 0.797 & 0.990 & 0.803 & 15.0 & 0.000 & 0.005 & 0.765 \\
\hline & 5.0 & 0.906 & 0.997 & 0.922 & 20.0 & 0.000 & 0.026 & 0.921 \\
\hline \multirow[t]{3}{*}{1.0} & 25.0 & 0.405 & 0.730 & 0.868 & 25.0 & 0.163 & 0.332 & 0.312 \\
\hline & 50.0 & 0.701 & 0.999 & 1.000 & 50.0 & 0.057 & 0.922 & 0.698 \\
\hline & 75.0 & 0.872 & 1.000 & 1.000 & 75.0 & 0.011 & 0.993 & 0.474 \\
\hline
\end{tabular}

Note: $A S W_{c}$ and $A S W_{t}$ denote with-constant and with-trend $A S W$, respectively.

Table 6. Number of frequencies selected by with-constant $M W$ and $A S W$ algorithms and BIC for volatility indices

\begin{tabular}{lccccc}
\hline Volatility index & $M W(5 \%)$ & $M W(10 \%)$ & $A S W(5 \%)$ & $A S W(10 \%)$ & BIC \\
\hline DJIA (VXD) & $0(0.92)$ & $2(0.76)$ & $2(0.76)$ & $2(0.76)$ & $0(0.92)$ \\
NASDAQ-100 (VXN) & $0(0.94)$ & $2(0.82)$ & $2(0.82)$ & $2(0.82)$ & $0(0.94)$ \\
S\&P 500 (VIX) & $0(0.92)$ & $2(0.76)$ & $2(0.76)$ & $2(0.76)$ & $2(0.76)$ \\
EuroCurrency (EVZ) & $1(0.90)$ & $1(0.90)$ & $1(0.90)$ & $3(0.85)$ & $0(0.97)$ \\
S\&P 100 (VXO) & $2(0.74)$ & $2(0.74)$ & $2(0.74)$ & $2(0.74)$ & $2(0.74)$ \\
FTSE 100 (VFTSE) & $2(0.81)$ & $2(0.81)$ & $2(0.81)$ & $2(0.81)$ & $0(0.95)$ \\
\hline
\end{tabular}

Note: Values of $\hat{\phi}$ are given in parentheses. 


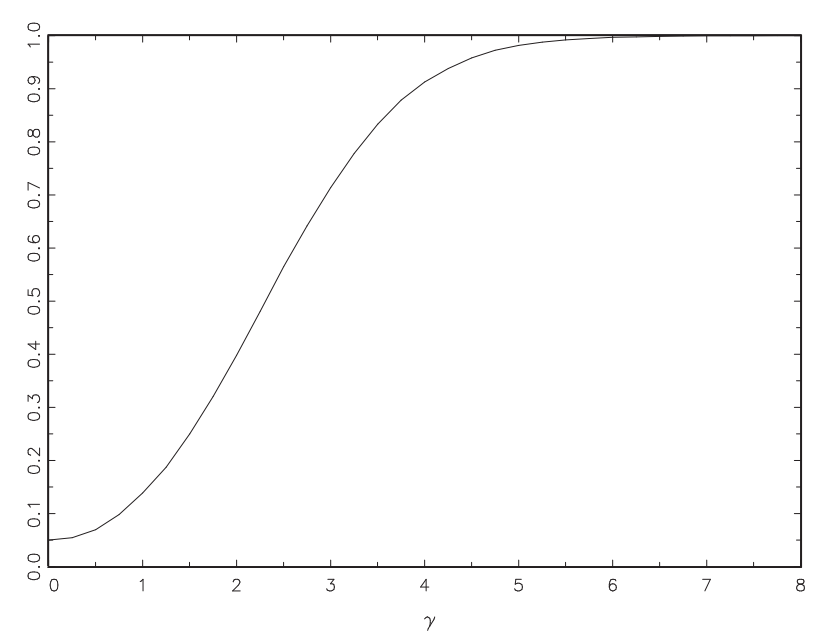

(a) I(0) shocks, $n=1$

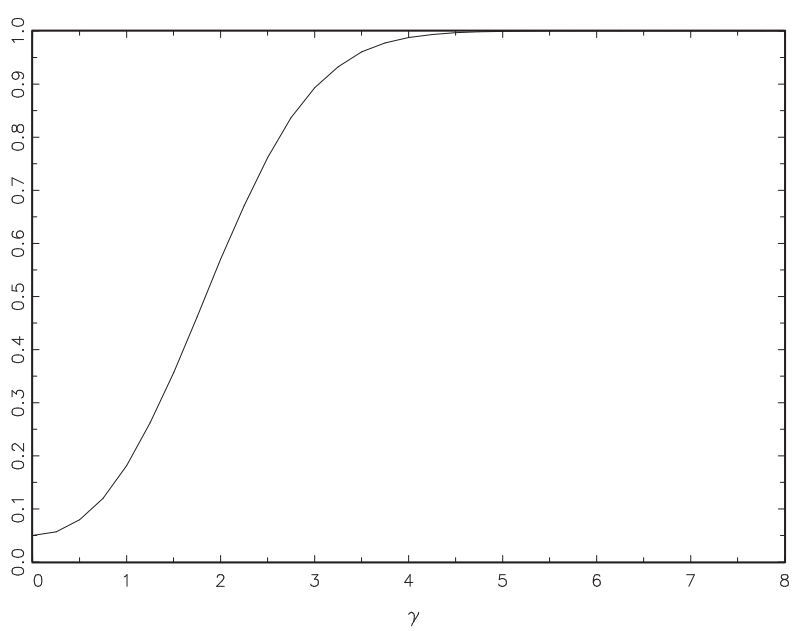

(c) I(0) shocks, $n=2$

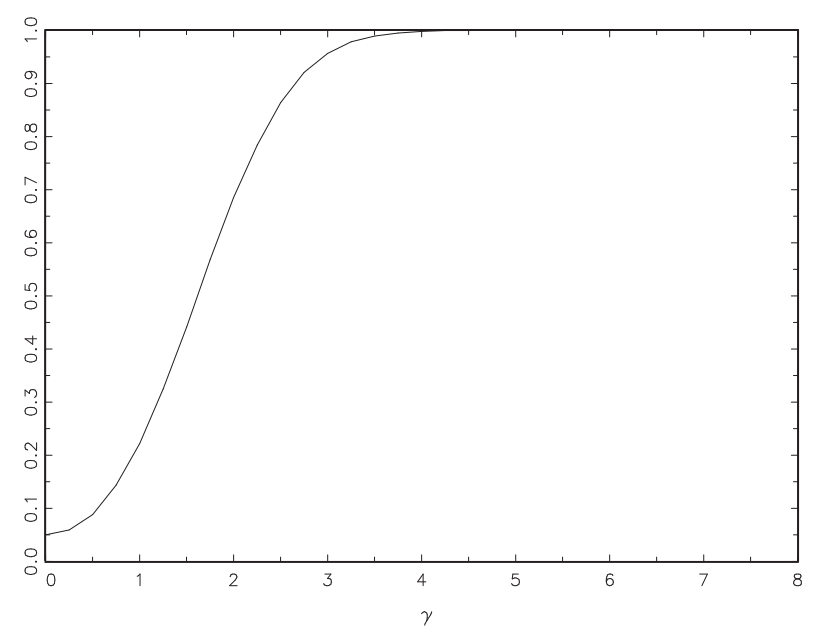

(e) I(0) shocks, $n=3$

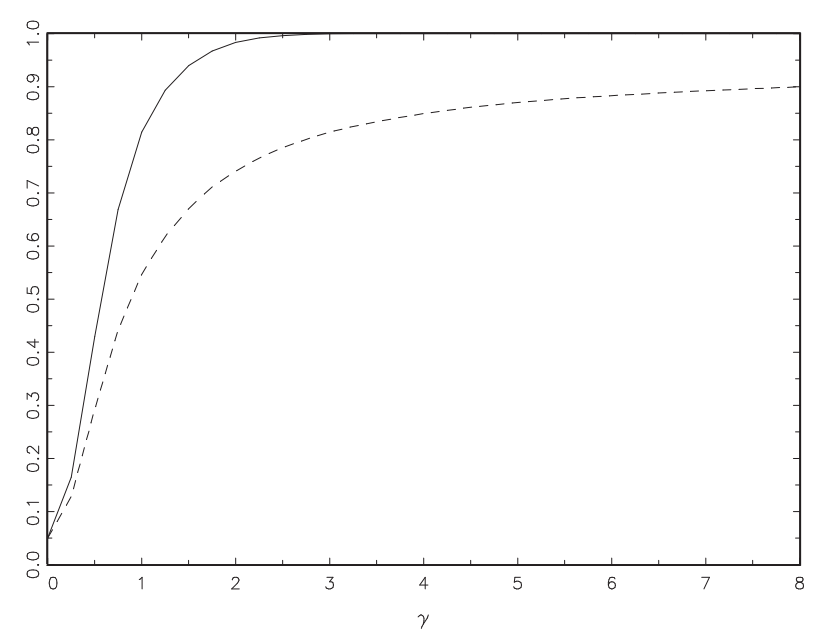

(b) I(1) shocks, $n=1$

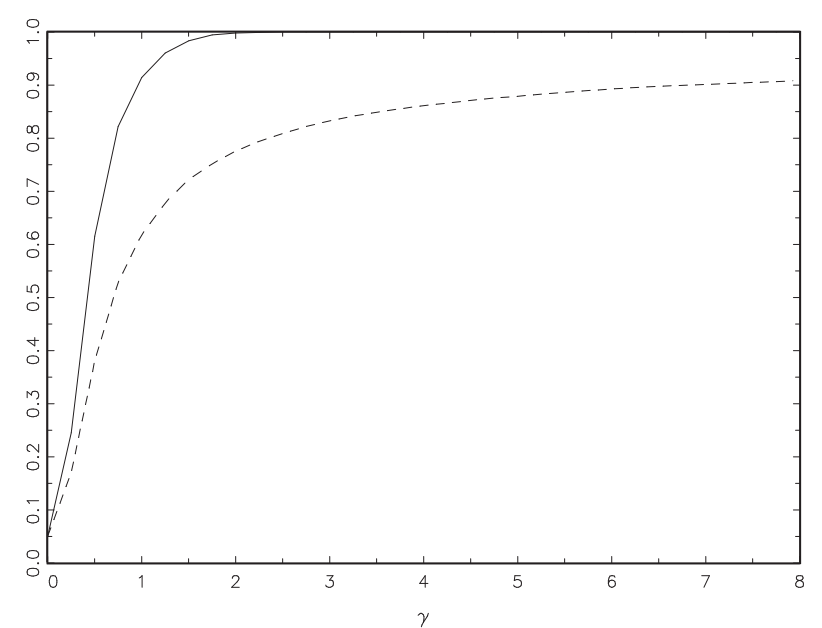

(d) I(1) shocks, $n=2$

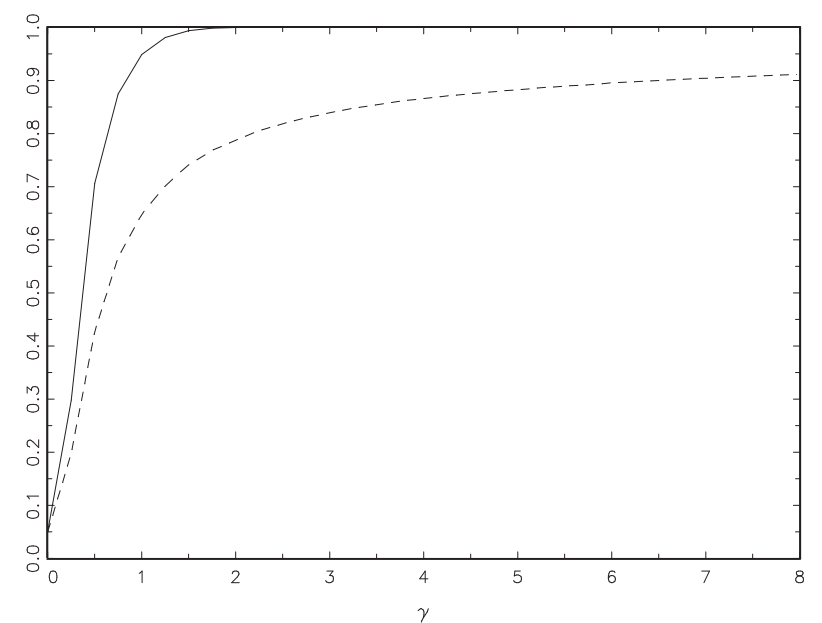

(f) I(1) shocks, $n=3$

Figure 1. Local asymptotic power of with-constant tests: $M W_{0}^{n}:---, A S W_{0}^{n}$ : 


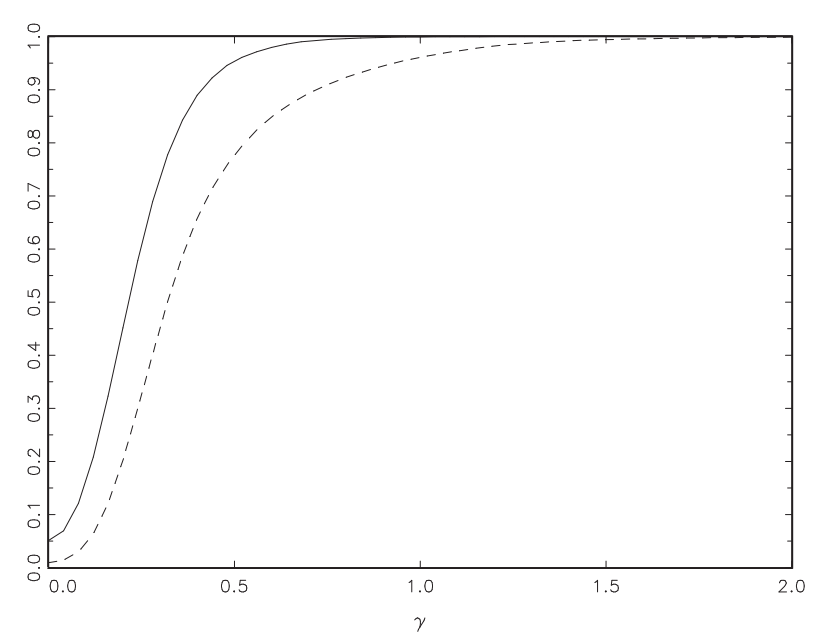

(a) $\phi=0.00$

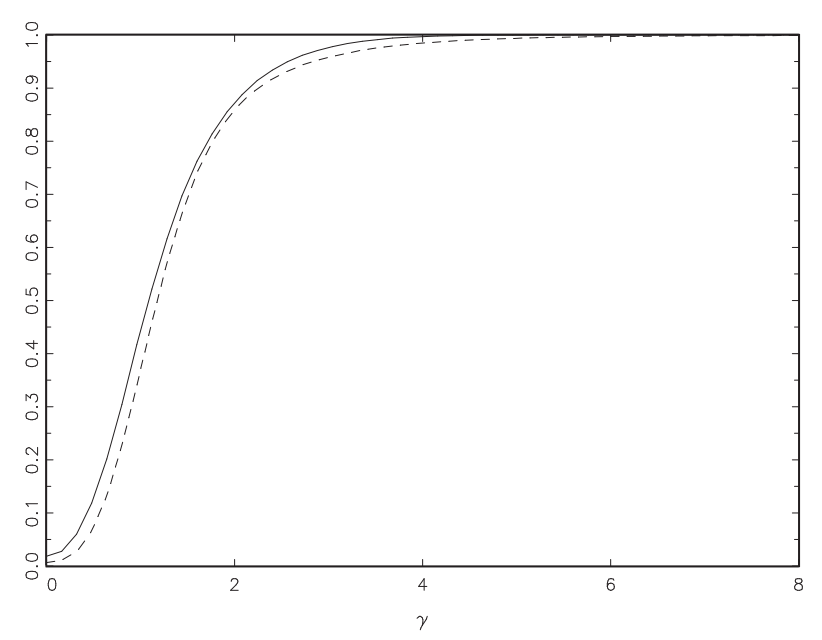

(c) $\phi=0.70$

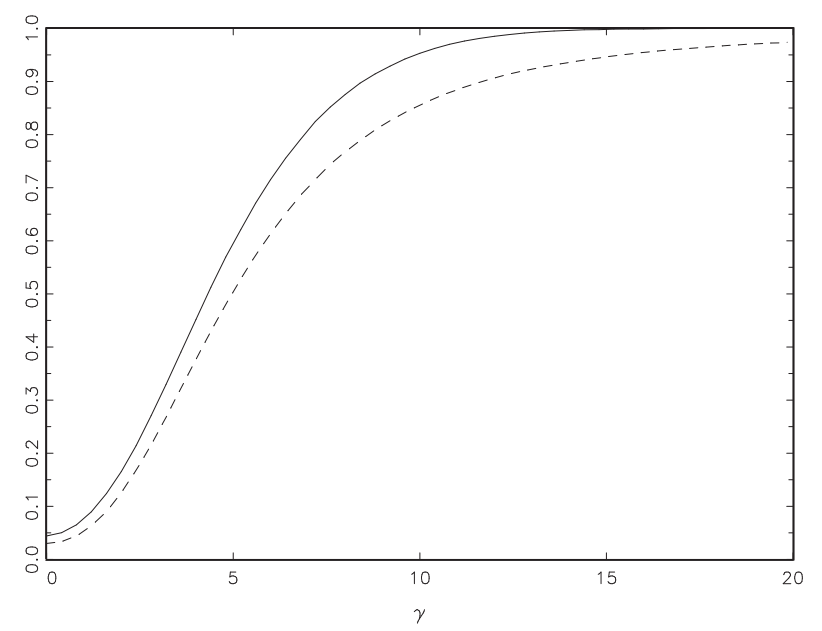

(e) $\phi=0.95$

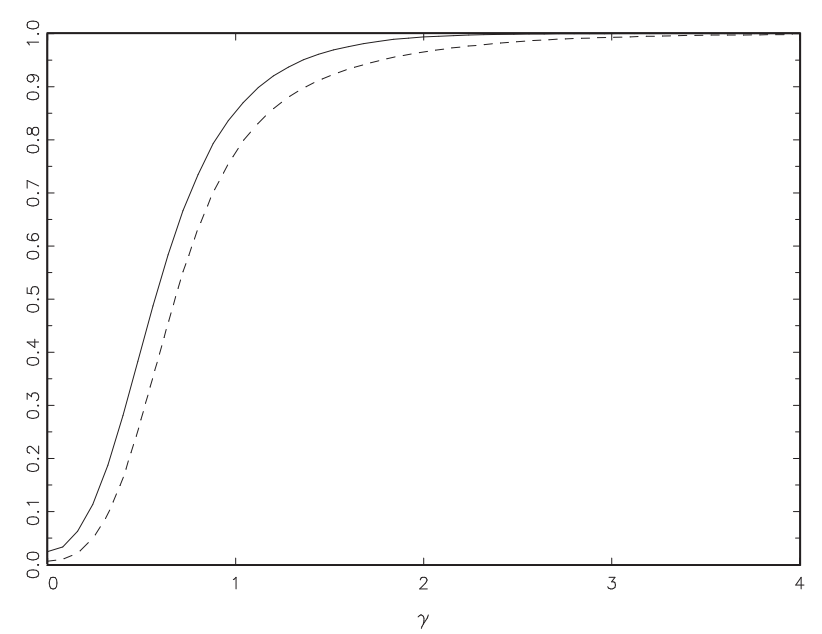

(b) $\phi=0.50$

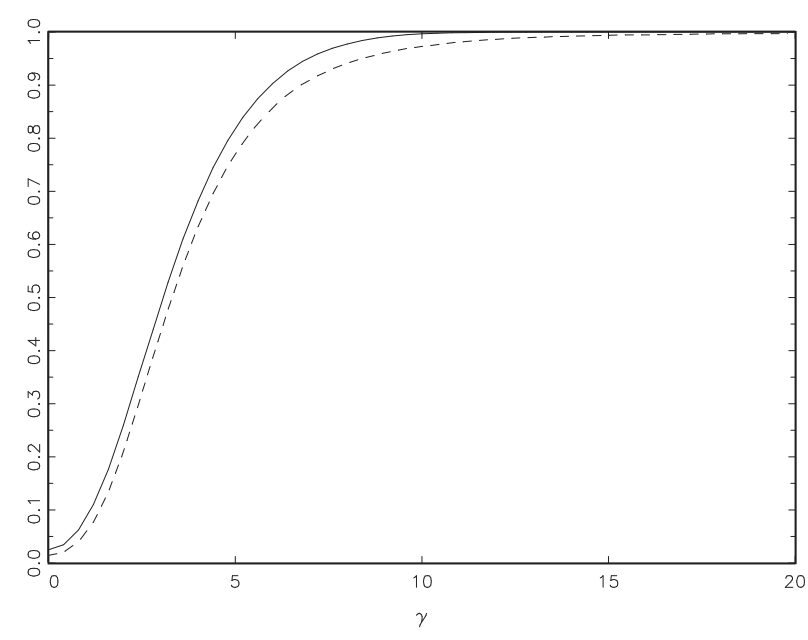

(d) $\phi=0.90$

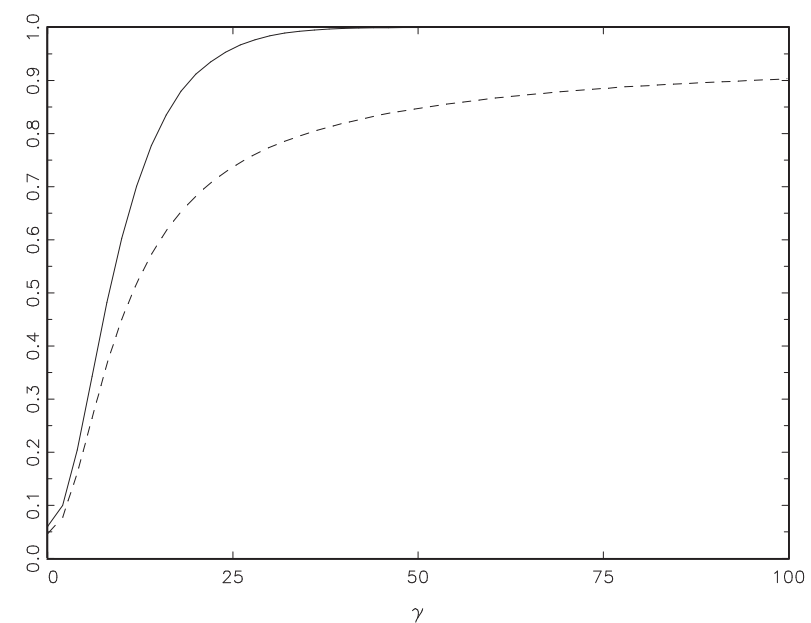

(f) $\phi=1.00$

Figure 2. Finite sample power of with-constant tests: $T=150, n=1 ; M W_{0}^{n}:---, A S W_{0}^{n}:-$ 


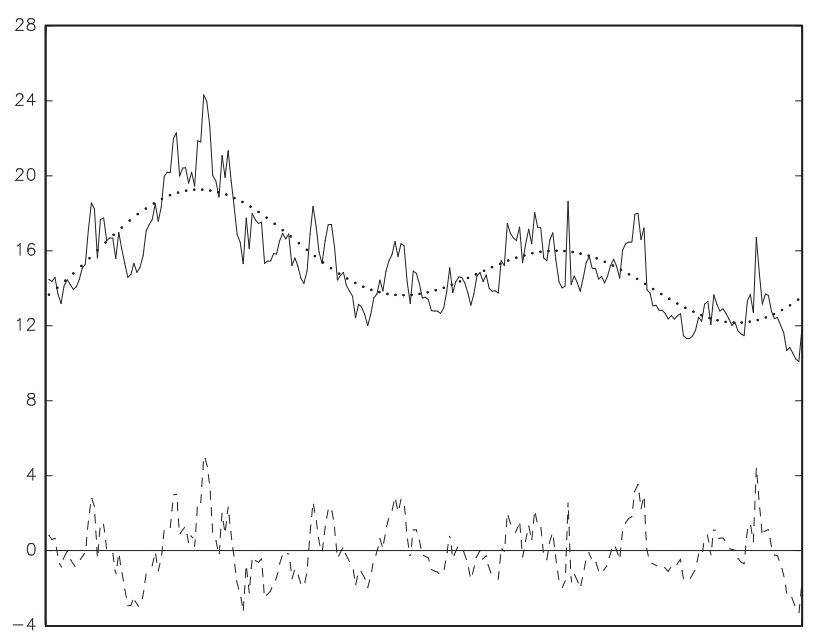

DJIA (VXD)

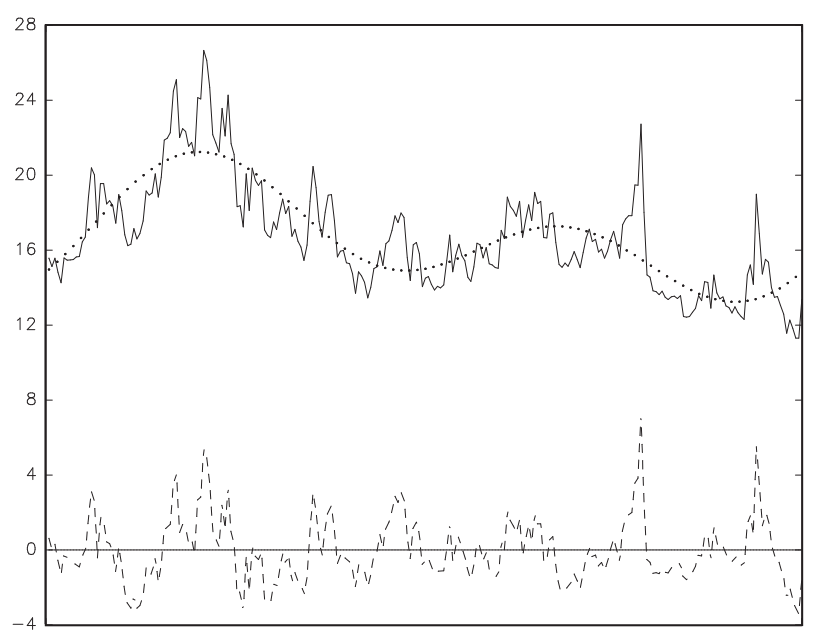

S\&P 500 (VIX)

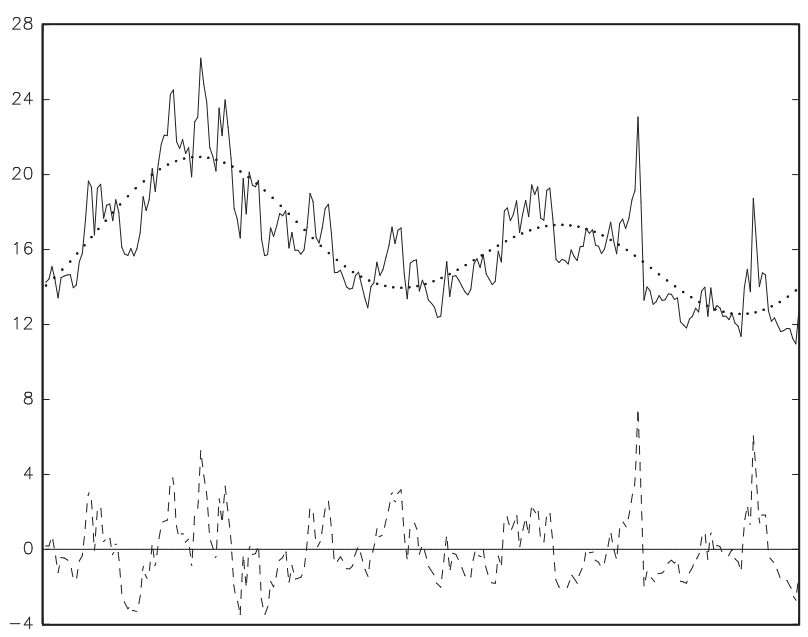

S\&P $100($ VXO)

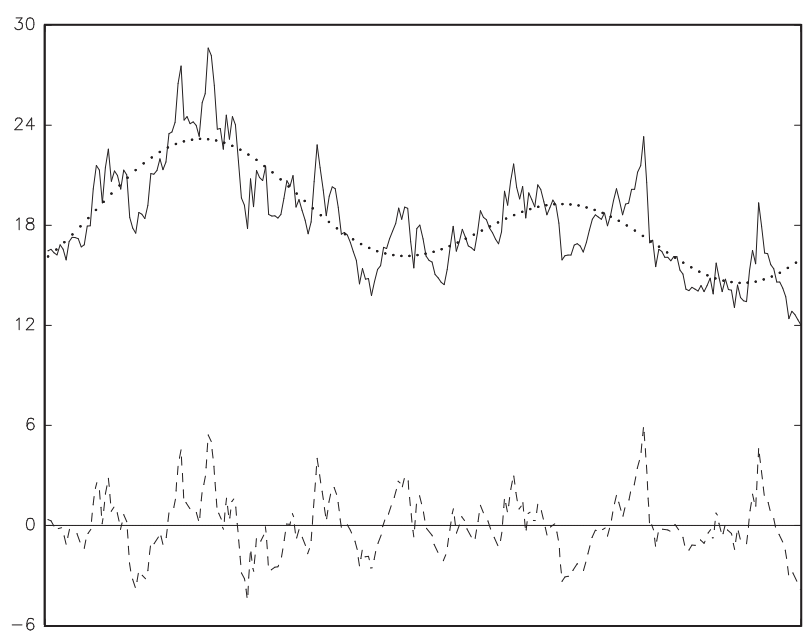

NASDAQ-100 (VXN)

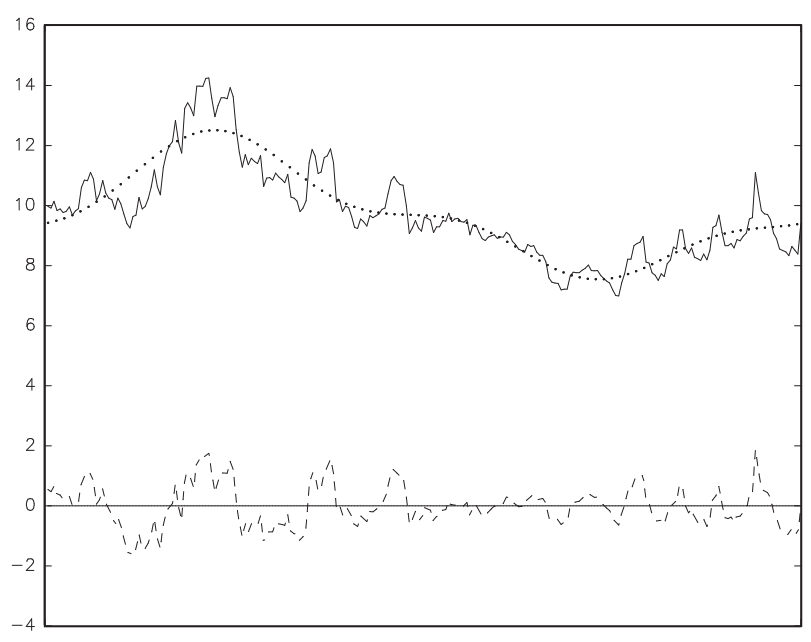

EuroCurrency (EVZ)

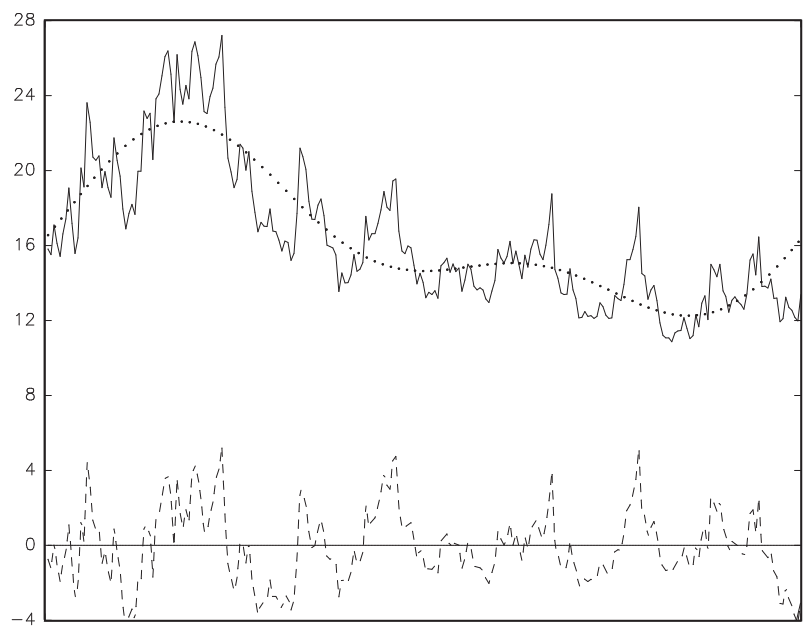

FTSE 100 (VFTSE)

Figure 3. Nonlinear deterministic components detected in volatility indices: Index: — , Fitted deterministic: $\cdots$, Detrended series: - - - 